\title{
Impact of the food matrix on iodine bioavailability
}

\author{
M. Redway, M. Bouga and E. Combet \\ Human Nutrition, School of Medicine, College of Medical Veterinary and Life Sciences, University of Glasgow, \\ Glasgow Royal Infirmary, Glasgow, G31 2ER, UK.
}

Iodine insufficiency is the most common cause of preventable mental impairment worldwide and can have significant adverse effects on thyroid function and child development. In the UK, there is neither an iodine fortification programme nor a current national monitoring programme for iodine status ${ }^{(1)}$. The main dietary sources of iodine in the UK are seafood and dairy products (especially milk) ${ }^{(2)}$, however, with recently reported iodine insufficiency in the $\mathrm{UK}^{(3)}$, other avenues of increasing dietary intakes of iodine should be investigated. Seaweed is not a popular food in Western diets, although it is rich in iodine and low-level supplementation has been shown to improve iodine status ${ }^{(4)}$.

In this pilot study, the impact of the food matrix of three iodine-rich foods (seaweed, fish and milk) on iodine bioavailability (as a \% of the dose ingested) was explored. A total of 11 participants were recruited to a randomised cross-over trial. All participants completed each of the three study arms, with a 2-day washout period between each arm. Participants were required to follow a low-iodine diet for the duration of the study. Urine was collected in time fractions $(-12-0 ; 0-1 ; 1-2 ; 2-3 ; 3-5 ; 5-8 ; 8-12 ; 12-24$ and $24-36$ hours) during the 12 hours preceding and the 36 hours following each meal for iodine level monitoring. Differences in excreted iodine between the 3 arms were measured using a one-way repeated measures ANOVA.

After 36 hours, the cumulated amounts of iodine excreted in urine following consumption of fish and milk were $86 \%$ (SD: $17 \%$ ) and $87 \%$ (SD: $39 \%$ ), respectively. In contrast, only $60 \%$ (SD: $16 \%$ ) of the iodine consumed in seaweed was excreted in urine after 36 hours. The cumulated amount of iodine excreted in urine was not statistically significantly different between the three study arms, possibly due to the high variability within and between participants $(p=0.08)$.

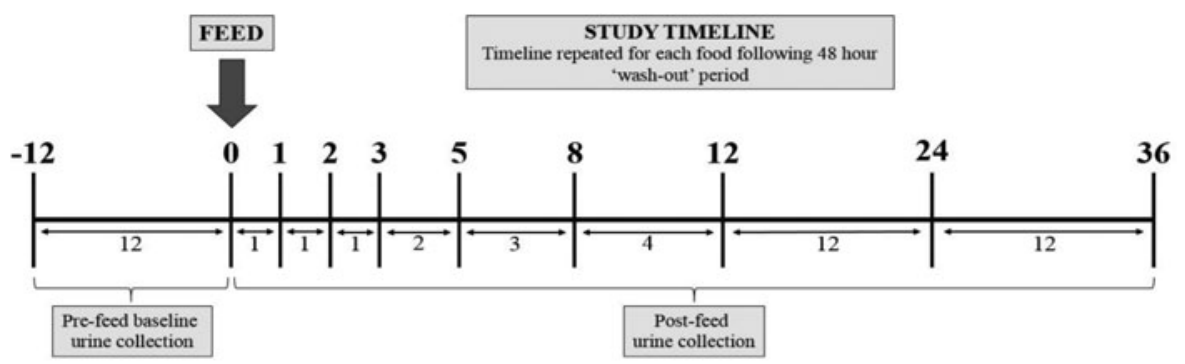

Iodine excretion from seaweed was more variable between participants and may reflect the difference in the food matrix of the seaweed compared to that of fish and milk, which may delay iodine release and absorption ${ }^{(4)}$. Further research is required to elucidate the specific mechanisms controlling iodine bioavailability in the three test foods. These pilot data suggest that the food matrix introduces an element of variability, which must be investigated further. All three foods, including seaweed, offer dietary options for iodine intake. Seaweed is also relevant for food fortification, as previously demonstrated, to improve the nutritional value of foods such as pizza, without negatively affecting either taste or appearance ${ }^{(5)}$.

1. Lazarus JH (2015) Eur Thyroid J 3, 3-6.

2. Haldimann M, Alt A, Blanc A et al. (2005) J Food Comp Anal 18, 461-471.

3. Vanderpump MPJ, Lazarus JH, Smyth PP et al. (2011) The Lancet 377, 2007-2012.

4. Combet E, Ma ZF, Cousins F et al. (2014) Br J Clin Nutr 112, 753-761.

5. Combet E, Jarlot A, Aidoo KE et al. (2013) Public Health Nutr 17, 2577-2586.

This study was conducted in collaboration with the British Broadcasting Corporation (BBC). 